\title{
Experimental and numerical investigations of switching wave dynamics in a normally dispersive fibre ring resonator
}

\author{
Bruno Garbin ${ }^{1}$, Yadong Wang ${ }^{1}$, Stuart G. Murdoch ${ }^{1}$, Gian-Luca Oppo ${ }^{2}$, Stéphane Coen ${ }^{1}$, and Miro Erkintalo ${ }^{1}$ \\ 1 The Dodd-Walls Centre for Photonic and Quantum Technologies, Department of Physics, The University of Auckland, \\ Auckland 1142, New Zealand \\ 2 SUPA and Department of Physics, University of Strathclyde, Glasgow G4 0NG, Scotland, EU
}

Received: date / Revised version: date

\begin{abstract}
Optical frequency combs generated in normally dispersive Kerr microresonators have been observed to correspond to dark temporal structures, and theoretically explained as interlocked switching waves (also known as domain walls or fronts). The time-domain dynamics that underpin the formation of this type of frequency combs has however so far eluded direct experimental observation. Here we use a closely related system - a synchronously driven optical fibre ring resonator — to experimentally study the dynamics of deterministically excited switching waves. We measure the switching wave velocities across broad parameter regions, and observe clear signatures of interlocking behaviour leading to the formation of persisting dark pulses. Our experimental findings are in good agreement with simulations of the mean-field Lugiato-Lefever equation, and strongly support the nature of normal-dispersion microresonator frequency combs suggested in the literature.
\end{abstract}

\section{Introduction}

Coherently-driven dispersive Kerr resonators have attracted considerable interest over the last ten years. On the one hand, macroscopic ring cavities constructed from standard single-mode optical fibres $[1,2]$ have been used for fundamental studies of dissipative patterns and localised structures known as temporal cavity solitons (TCSs), which can be applied as bits in all optical buffers [3-9]. On the other hand, monolithic microresonators have enabled the generation of optical frequency combs [10-18] whose unique and attractive characteristics make them ideally suited for applications such as telecommunications $[19,20]$ and spectroscopy $[21,22]$.

Besides scaling factors associated with the differences in physical size, microresonators and fibre ring resonators display very similar dynamics $[4,23,24]$. In fact, both systems can be described by the very same mean-field equation [23, 25] that is analogous to the celebrated Lugiato-Lefever equation (LLE) of spatially diffractive cavities [26], initially used to describe driven plasmas [27-29] and a forced condensate [30]. This similarity has, in particular, allowed fibre ring resonator experiments to provide invaluable insights into comb dynamics that cannot be resolved in microresonators due to their small size [31-33]. So far, the vast majority of studies have focused on resonators that exhibit anomalous dispersion (at the driving wavelength), arguably because the modulational instabilities (MI) that underpin the formation of cavity solitons and optical frequency combs manifest themselves over a much wider (and in general more acces- sible) range of parameters compared to resonators exhibiting normal dispersion $[34,35]$. The difficulty of achieving anomalous dispersion in certain key regions of the electromagnetic spectrum has, however, also prompted research into better understanding the dynamics and opportunities in the normal dispersion regime, especially in the context of microresonator frequency combs [36-41]. Of particular significance has been the realisation that higher-order effects can considerably expand the range of parameters over which MI can occur, thus rendering the creation of frequency combs feasible in the normal dispersion regime $[8,40-42]$. Indeed, Xue et al have convincingly demonstrated that, normal dispersion notwithstanding, interactions between different mode families can alter the phase of spectral components around the driving wavelength, permitting phase-matching of the four-wave mixing that underlies both MI and the subsequent formation of broadband frequency combs $[40,41]$.

A remarkable finding reported in $[40,41]$ is that, following the initial MI activated by higher-order effects, the intracavity field may evolve into a fully phase-locked state corresponding to dark pulses - localised low-intensity dips - embedded in a high-intensity continuous-wave (cw) background. Several theoretical models have been put forward to explain the physical essence of these dark structures $[40,43,44]$. Parra-Rivas et al made the particularly convincing case that the dark structures consist of two interlocked switching waves (SWs) [44]. SWs, also known in the literature as domain walls or fronts, are travelling front solutions connecting the two homoge- 
nous states of a bistable system [45]. They have been extensively investigated in many different contexts such as chemical reactions [46], biological [47] and magnetic systems [48], granular media [49], hydrodynamics [50], population dynamics [51], flame front propagation [52], as well as nonlinear optics $[45,53-60]$.

Under purely diffusive transverse coupling, SWs have hyperbolic tangent profiles and they travel until the more stable state has fully invaded the less stable one [61]. There exists only one particular set of parameters - known as the Maxwell point - at which the fronts remain stationary. In the presence of dispersion or diffraction, however, SWs can display oscillatory tails [62], which allow two travelling SWs to interact and trap each other (akin to the formation of soliton bound states $[63,64])$. This results in the arrest of their relative motion, thereby giving rise to a stable localised region of one state embedded in the other [54,62, 65-68]. Before recently attracting attention in the context of frequency comb generation in dispersive nonlinear optical resonators, such front locking dynamics have been extensively studied in spatially diffractive systems with one and two transverse dimensions [45, 54,65].

As demonstrated by Parra-Rivas et al (based on theoretical analysis of the LLE), interlocked SWs can qualitatively explain many of the salient features observed in experiments involving frequency comb generation in normally dispersive microresonators [44]. However, the small physical size of microresonators obstructs quantitative study of the transient SW dynamics and their behaviour at the point of interlocking. Macroscopic fibre ring resonators are devoid of this obstruction. They should therefore permit more controlled investigations but so far only steady-state SW observations have been reported [53].

In this Article, we experimentally and numerically study the transient dynamics of deterministically excited time-domain SWs and their interlocking. Specifically, we use a synchronously-driven, normally dispersive fibre ring resonator, and demonstrate robust SW excitation by intensity modulation of the flat-top nanosecond pulses driving the resonator. By monitoring the transient SW dynamics in real time, we are able to experimentally measure the SW velocity across a wide range of parameters, as well as the transient build-up of interlocked states. Our experiments are in good agreement with simulations of the LLE, and they strongly corroborate the proposition that mode-locked frequency combs in normally dispersive microresonators correspond to interlocked SWs.

\section{Illustrative numerical simulations}

We begin by briefly recalling the physics of SWs and their interlocking behaviour. To this end, we present results from numerical simulations pertinent to the experiments that will follow.

It is well known that, in the limit of high cavity finesse, the dynamics of a coherently driven fibre ring resonator is governed by a mean field equation that is formally identical to the celebrated Lugiato-Lefever equation of spatially diffractive Kerr cavities $[1,26]$. Adopting the same normalisation as in [4], the equation reads:

$$
\frac{\partial E(t, \tau)}{\partial t}=\left[-1+i\left(|E|^{2}-\Delta\right)-i \eta \frac{\partial^{2}}{\partial \tau^{2}}\right] E+\sqrt{X}
$$

Here the variable $t$ corresponds to a "slow" time that describes the evolution of the electric field envelope $E(t, \tau)$ inside the resonator at the scale of the cavity photon lifetime, while $\tau$ corresponds to a "fast" time defined in a reference frame moving at the group-velocity of light at the driving wavelength and describes the temporal profile of the intracavity field envelope. The terms on the right-hand side of Eq. (1) describe - from left to right - the cavity losses, the Kerr nonlinearity, the detuning between the driving frequency and the closest cavity resonance ( $\Delta$ is the detuning parameter), second-order group-velocity dispersion ( $\eta$ is the sign of the group-velocity dispersion coefficient $\beta_{2}$ ), and the coherent driving of the cavity ( $X$ represents the driving power). Noise has not been included in the present description since we are interested in deterministic dynamics of SWs. We also note that in our simulations we make use of periodic boundary conditions.

The homogeneous (cw) stationary solutions of Eq. (1) satisfy the well-known cubic polynomial of dispersive optical bistability [1]:

$$
X=Y^{3}-2 \Delta Y^{2}+\left(\Delta^{2}+1\right) Y,
$$

where $Y=|E|^{2}$ represents the intracavity power. For constant driving power $X$, these solutions describe a single Lorentzian cavity resonance that is tilted due to the Kerr nonlinear phase shift, and which can be expressed as

$$
\Delta=Y \pm \sqrt{\frac{X}{Y}-1}
$$

Figure 1(a) shows an example of such a tilted cw resonance, evaluated using parameters similar to the experiments that will be described in Section 3. As is well known [and can be seen in Fig. 1(a)], the cw solutions exhibit bistability for detunings between the up- and downswitching points $\Delta_{\uparrow}$ and $\Delta_{\downarrow}$, respectively (for the parameters of Fig. $1(\mathrm{a}), \Delta_{\uparrow}=4.9$ and $\left.\Delta_{\downarrow}=19.5\right)$. Note that the intermediate branch [dashed red line in Fig. 1(a)] is unconditionally unstable, and will not be discussed further.

In the regime of anomalous group-velocity dispersion $(\eta=-1)$, the upper branch of the cw solutions exhibits MI over a wide region of parameters, and this process underpins the formation of Kerr optical frequency combs and TCSs $[4,18]$. In contrast, we are interested here in normally dispersive cavities $(\eta=+1)$, where the two cw states are simultaneously stable across most of the bistable region (MI is limited to a small region around $\Delta_{\uparrow}$ at the end of the lower cw branch, and is not accessed in our experiments). In this case, both the lower and upper $\mathrm{cw}$ states can persist indefinitely (i.e. in steady-state), and coexist simultaneously at different positions along the resonator. Specifically, when operating in the bistable region, the temporally extended intracavity field can be composed 
of finite domains associated with the two different cw states. Because of the dispersive coupling, the domains are connected by fronts, or "switching waves," featuring a smooth edge and an oscillatory tail $[44,54,69]$, as illustrated in Fig. 1(b). (Note that, due to periodic boundary conditions, there are two such SWs simultaneously present, but only one is shown in Fig. 1(b) for clarity.) In general, however, the $\mathrm{cw}$ states only co-exist for a limited time: the SWs move at a constant velocity, causing one type of domain to invade the other (one of the cw states can thus be considered as metastable [50]). The velocity of the SWs, as well as the relative stability of the two different cw states, depend on the control parameters $[44,65,70]$. For fixed driving power $X$, one can qualitatively appreciate that the lower (upper) cw state is more stable for detunings close to the down- (up-) switching point. It is only for one special set of parameters, known as the Maxwell point, that the two cw states are "equally" stable and can co-exist indefinitely in steady-state. For this set of parameters, the SWs exhibit of course zero velocity.

To illustrate this description, we show in Figs 1(c)-(e) results from numerical simulations of the LLE that mimic the experiments that will follow. Here, we use a "space-
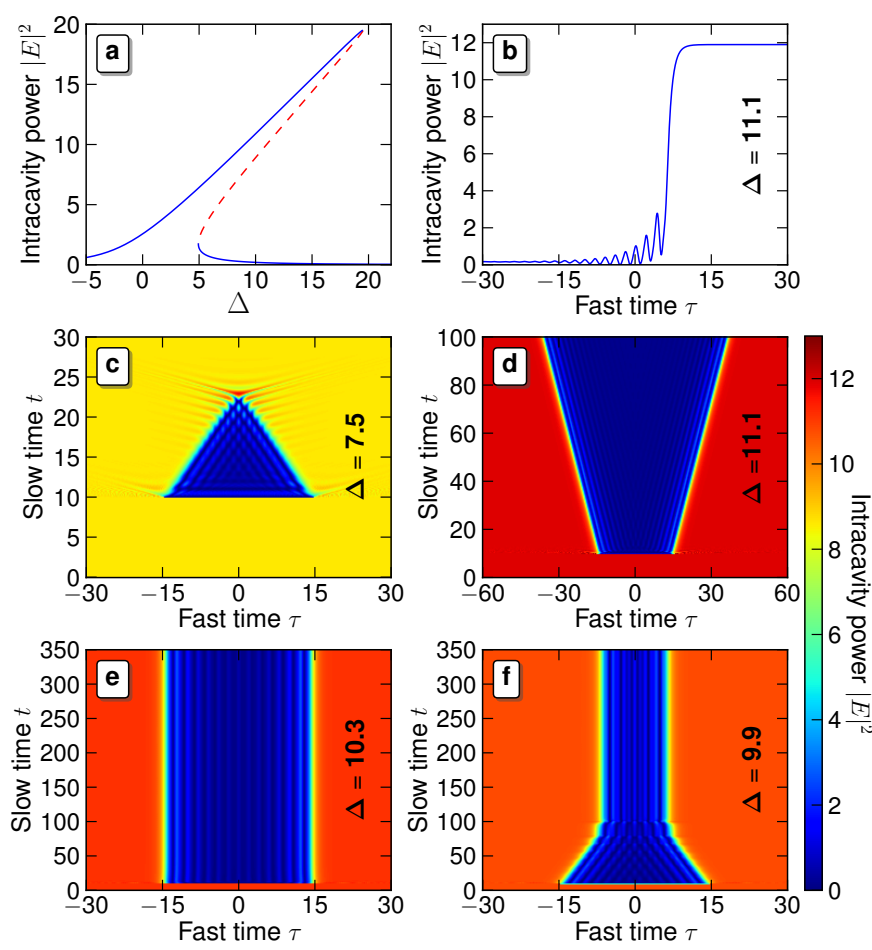

Fig. 1. Results of numerical simulation of the normalised LLE, obtained for a set driving power $X=19.5$. (a) Intracavity power of cw solutions as a function of cavity detuning $\Delta$. (b) Temporal power profile of a typical SW for $\Delta=11.1$. (c)-(f) Pseudo-colour plots showing the evolution (bottom to top) of the intracavity power obtained for detunings $\Delta$ as shown in each panel. time" representation that shows how the fast-time profile of the intracavity power (horizontal) evolves with slowtime (bottom to top), for a fixed driving power $X=19.5$ and for a range of detuning values $\Delta$. In each case, the initial condition consists of the upper cw state, which is then perturbed - at slow time $t$ equal to 10 - by a superGaussian intensity dip that locally approximates the lower cw state. As seen in Fig. 1(c), for a comparatively small $\Delta=7.5$, the upper cw state quickly invades the lower one, while the opposite is true for a larger $\Delta=11.1$ [Fig. 1(d)]. For the driving power used in Fig. 1, the Maxwell point occurs at $\Delta_{\mathrm{M}} \approx 10.3$, and indeed, our simulation reveals stationary SWs in this condition [Fig. 1(e)].

Although the SWs are unconditionally stationary only at the Maxwell point, it is also possible for two SWs to "lock" in a finite range of parameters around the Maxwell point through interactions mediated by their oscillatory tails. Since oscillations are only present near the lower edge of the SWs [Fig. 1(b)], this can only happen for a low intensity domain embedded in a high intensity background: the result is a dark localised structure (bright structures become possible e.g. in presence of high-order dispersion or nonlocality [44]). In Fig. 1(f), we illustrate such interlocking numerically with $\Delta=9.9(X=19.5$ as before). As clearly seen, the two approaching SWs eventually come to a halt. It is precisely this interlocking behaviour that has recently been proposed to underlie the formation of mode-locked Kerr frequency combs in normally dispersive microresonators $[44,71]$.

For a given set of parameters, the SWs can lock for several discrete separations, corresponding to localised structures of different widths. Such multistability can be physically understood as locking between distinct cycles of the oscillatory SW tails, and is associated with a bifurcation structure referred to as collapsed snaking $[69,71]$. Because the tail oscillations of the SWs are exponentially damped, one finds that structures with small widths generally exhibit larger regions of existence and stability $[44,72]$. Notwithstanding, the particular structures observed in simulations and experiments are not limited to those with the smallest widths, but rather are selected on basis of the initial condition (or the external perturbation) used to excite the SWs. For example, the final structure observed in Fig. 1(f) corresponds to the largest stable separation for two SWs for the chosen parameter values (here the SW separation $\Delta \tau=12$ in normalised units). It is representative of the experimental observations that we present below, and in which the limited temporal resolution of our measuring apparatus prevents us from observing the shortest structures.

\section{Experimental setup}

In this work, our aim is to experimentally investigate the temporal SW dynamics described above. To this end, we use the setup illustrated in Fig. 2. It consists of a passive fibre ring resonator with a total length of about 90 metres. The cavity is mainly constructed of a dispersion shifted fibre (Corning MetroCor, 88 metres long) 


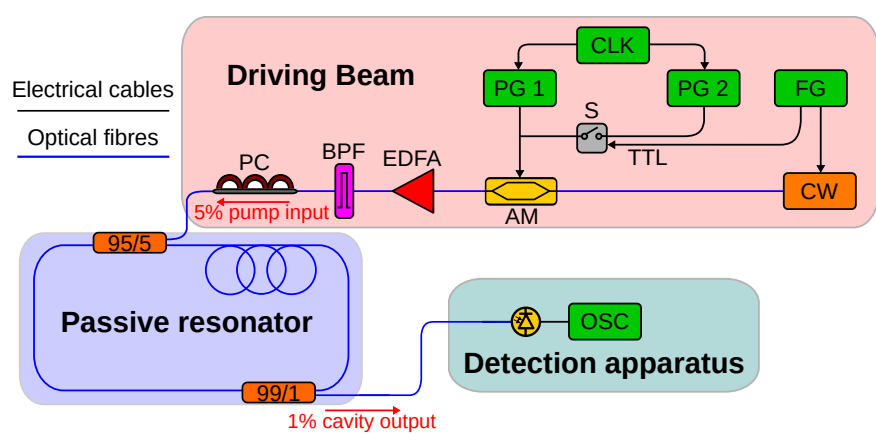

Fig. 2. Schematic illustration of the experimental set-up. cw, tunable $\mathrm{cw}$ driving laser; FG, function generator; PG 1 and PG 2, pattern generators as described in the text; CLK, clock; S, electrical switch; AM, Mach-Zehnder intensity modulator; EDFA, Erbium-doped fibre amplifier; BPF, band-pass filter; $\mathrm{PC}$, polarisation controller; OSC, oscilloscope.

that exhibits normal dispersion $\left(\beta_{2}=8.1 \mathrm{ps}^{2} / \mathrm{km}\right)$ at the driving wavelength of $1550 \mathrm{~nm}$, but also includes about $0.7 \mathrm{~m}$ of standard SMF-28 fibre with anomalous dispersion $\left(\beta_{2}=-21.4 \mathrm{ps}^{2} / \mathrm{km}\right)$. This yields a round-trip time $t_{\mathrm{R}}$ of about $430 \mathrm{~ns}$, corresponding to a free-spectral-range (FSR) of $2.3 \mathrm{MHz}$, and a cavity averaged dispersion of $\left\langle\beta_{2}\right\rangle=7.9 \mathrm{ps}^{2} / \mathrm{km}$. The resonator also includes a $95 / 5$ coupler used to inject the driving field into the cavity, as well as a 99/1 coupler through which the intracavity dynamics are monitored using a $12.5 \mathrm{GHz}$ photodetector connected to a real time oscilloscope. Overall, the cavity has a high measured finesse of 37 , corresponding to a photon lifetime of about 6 round trips.

To drive the cavity, we use flat-top, quasi-cw, nanosecond pulses at a repetition rate synchronised to the cavity FSR $[8,33,34,73]$. These pulses are obtained by intensity modulating a commercial, wavelength-tunable, Erbium-doped distributed feedback cw fibre laser (Koheras AdjustiK ${ }^{\mathrm{TM}}$ E15) by means of a Mach-Zehnder modulator (JDSU, $10 \mathrm{~Gb} / \mathrm{s}$ intensity modulator) driven by a $10 \mathrm{GHz}$ pattern generator (PG 1 in Fig. 2). After the modulator, the pulses are amplified up to $1.3 \mathrm{~W}$ peak power (corresponding to a normalised driving power $X=19.5$ ) using an Erbium-doped fibre amplifier (Keopsys $5 \mathrm{~W}$ ), and spectrally filtered to remove amplified spontaneous emission. A polarisation controller is finally used to set the state of polarisation of the driving pulses before their injection into the cavity.

The reason for driving the cavity with nanosecond pulses (instead of a purely cw field) is threefold. Firstly, pulses can be readily amplified to larger peak powers than $\mathrm{cw}$ fields, allowing access to larger resonance tilts and therefore larger detunings [33,73]. Secondly, the large bandwidth of the driving pulses suppresses the detrimental effects of stimulated Brillouin scattering (SBS). We note that previous fibre resonator studies involving TCSs in the anomalous dispersion regime relied on an intracavity isolator to mitigate SBS $[4,6,7]$. This method is not applicable to our experiment because, in contrast to TCSs which always sit on a lower state background, parts of our intracavity field are in the upper $\mathrm{cw}$ state, with

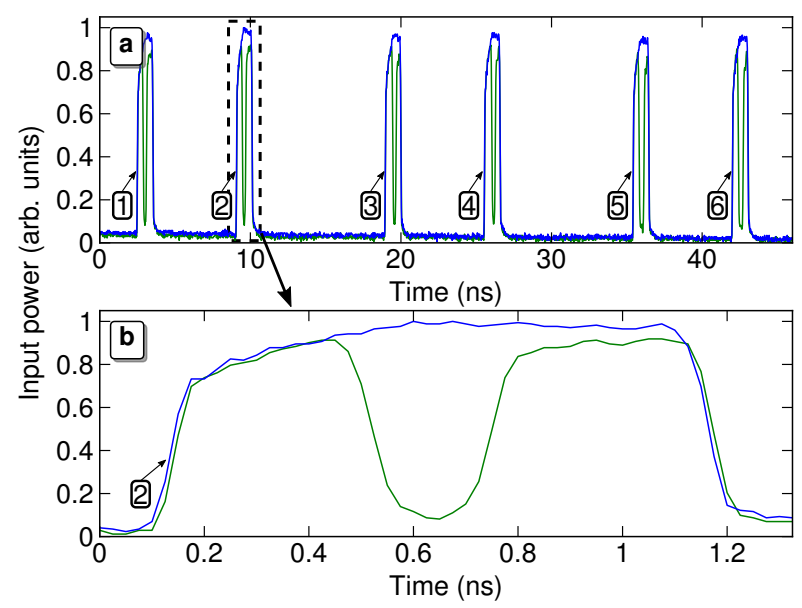

Fig. 3. (a) Pattern of quasi-cw pulses used to drive the resonator. The pattern repeats every $430 \mathrm{~ns}$, matching the cavity round-trip time, thus ensuring synchronous driving. Each pulse is labelled numerically to facilitate the discussion of our results (the labels match those in Fig. 5). (b) Enlarged view of driving pulse \#2. Blue (green) curves show the driving pulses in the absence (presence) of the perturbations used to excite SWs.

an intensity level much more susceptible to SBS. On the plus side, the lack of an isolator enables us to reach comparatively higher cavity finesses. Thirdly, by driving the cavity with a pulse pattern consisting of multiple widelyspaced nanosecond pulses per round trip (the whole pattern being synchronised to the cavity FSR), we can simultaneously realise several independent experimental realisations. The driving pattern used in our experiment contains six nanosecond-duration pulses and is illustrated in Fig. 3(a) [blue curve]. As can be seen, small differences in peak power levels exist between the different driving pulses due to modulation and amplification imperfections. This causes small artefacts in our observations which will be discussed below.

Because the nanosecond driving pulses are much longer than the picosecond-scale SWs we are interested in, the driving field can be considered as quasi-cw. We note, however, that a small synchronisation mismatch between the repetition rate of the driving pattern and the cavity FSR is generally unavoidable and results in an effective convective drift [53] that can hinder the interpretation of the data. In particular, the front expansion/contraction dynamics can appear asymmetric if examined in a (natural) reference frame fixed to the driving pattern repetition rate. To alleviate this issue, all our results are presented in a reference frame fixed to the cavity FSR, in which the SW motion is symmetric. (Of course, in this reference frame, a synchronisation mismatch causes the nanosecond background to exhibit a uniform temporal drift.)

Finally, to systematically excite SWs, we use a signal from a second pattern generator (PG 2 in Fig. 2) synchronised to the driving pattern to perturb selected driving pulses. That signal is made up of approximately Gaussian pulses, shorter than the driving pulses, and generated with 
a $\pi$ phase shift with respect to that of PG 1 . When fed through the electrical switch $(\mathrm{S})$, it thus effectively carves dips in the nanosecond driving pulses. The green curve in Fig. 3(a) illustrates the resulting perturbed driving pulse pattern [with an enlargement of one the pulses in Fig. 3(b)] compared to the unperturbed driving (blue curve). Note that the switch is only open during the initial phase of SW excitation and is blocked for the rest of the experiment. This effectively mimics the procedure used to excite SWs in the simulations presented in Fig. 1.

\section{Experimental results}

To investigate SW dynamics in a systematic way, we start each measurement by tuning the frequency of the driving laser with a function generator in order to select a detuning value $\Delta$ that lies in the regime of cw bistability. Specifically, we ramp up the detuning across the cavity resonance and onto the upper branch, i.e. from left to right in Fig. 1(a), and stop at a selected point of interest. In this way, the intracavity field initially corresponds to the upper $\mathrm{cw}$ state. Next, we perturb the driving pulses
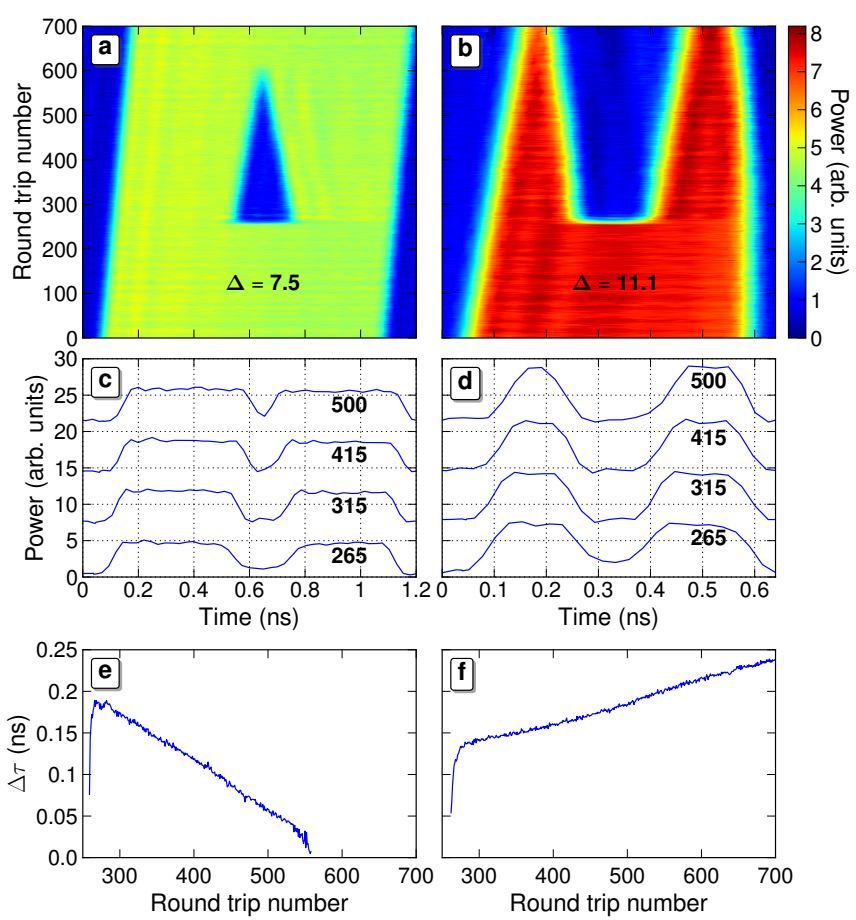

Fig. 4. Experimental measurements of SW dynamics, obtained for two different values of the cavity detuning as indicated. (a, b) Space-time diagrams showing the evolutions of the temporal profile of the cavity output power over 700 cavity round trips. The SWs are excited starting from round trip \#253. (c, d) Snapshots of the cavity output power profile at specified round trips, after excitation of the SWs. (e, f) Corresponding evolution of the temporal separation of the two SWs, $\Delta \tau$. for about 10 cavity round trips (with the S switch, as described above), which locally switches the intracavity field to the lower cw state, creating SWs on both sides. After the switch is returned to its original off position, and the perturbation has died off, the driving pulses regain their original flat-top shape (blue curve in Fig. 3) and the SWs are left to evolve unperturbed. The excitation and evolution of the SWs are recorded as a long time trace of the cavity output power on an oscilloscope.

Typical experimental results, obtained for two different cavity detunings, are shown in Figs 4(a) and (b). Here we visualise the intracavity field evolution by means of the same space-time representation as used in Figs 1(c)-(f), and that is obtained by (i) slicing a single long oscilloscope trace into segments separated by the cavity roundtrip time and (ii) concatenating the individual segments atop each other. Note that, as explained above, the visible drift of the nanosecond background pulse originates from a mismatch between the repetition rate of the driving pattern and the cavity FSR. As can be seen, for a small detuning $\Delta=7.5$ [Fig. 4(a)], the domain of lower cw state (induced by the applied perturbation) contracts at the expense of the surrounding upper state, with the two SWs moving towards each other. In contrast, for a larger detuning $\Delta=11.1$, the lower state domain expands, with the SWs moving away from each other [Fig. 4(b)]. In Figs 4(c) and $(d)$, we show corresponding snapshots of the oscilloscope traces at four different round trips, as indicated in the panels.

The results shown in Figs 4(a)-(d) clearly illustrate the motion of SWs. To obtain more quantitative insights, we show in Figs 4(e) and (f) the evolution of the temporal separation between the SWs, $\Delta \tau$. That quantity represents the temporal duration of the lower $\mathrm{cw}$ state domain and is extracted from our data as $\Delta \tau=\tau_{2}-\tau_{1}$, where $\tau_{2}$ $\left(\tau_{1}\right)$ is the temporal position of the trailing (leading) SW. We can clearly see that the domain size changes linearly over time, i.e. the SW moves at constant velocity. By extracting the slope of the curve, we can readily obtain a quantity that characterises the SW velocity,

$$
V=\frac{1}{2} \frac{\mathrm{d} \Delta \tau}{\mathrm{d} t / t_{\mathrm{R}}}
$$

where the factor of two accounts for the symmetric motion of the two SWs. For the measurements in Figs 4(a,c) and $(\mathrm{b}, \mathrm{d})$, we obtain $V \approx-0.29$ ps per round trip and $V \approx+0.13$ ps per round trip, respectively. Note that, with our definitions, $V<0(V>0)$ implies contraction (expansion) of the lower cw state, i.e. the SWs moving towards (away from) each other.

We have repeated the measurements over a wide range of cavity detunings, and summarise our findings in Fig. 5. Here we plot the measured SW velocities as a function of the detuning, with the different curves corresponding to the different pulses of the driving pattern (coloured curves with labels coinciding with those in Fig. 3). We believe that slight variations in the peak power levels of the different driving pulses (see Section 3) explain the small spread of SW velocities observed in Fig. 5. This spread is 


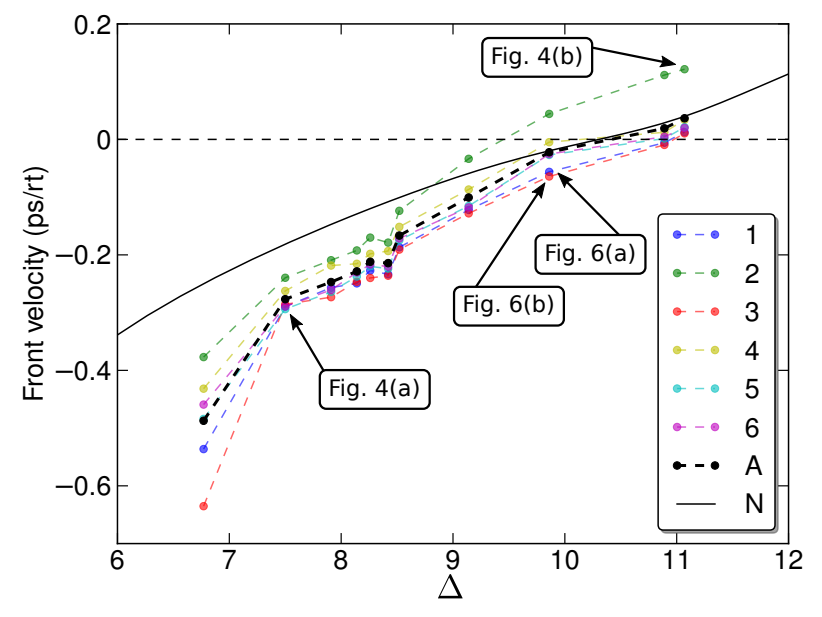

Fig. 5. Experimentally measured SW velocities as a function of cavity detuning $\Delta$. The different numerically-labelled curves correspond to the different driving pulses shown in Fig. 3(a). The black dashed curve (A) is the average over all experimental realisations. The solid black curve $(\mathrm{N})$ is the numerical prediction. Points corresponding to data displayed in other figures are labelled accordingly.

thus representative of the uncertainty in the measurement. To facilitate our analysis, we also plot in Fig. 5 the average SW velocity computed across all the different driving pulses (dashed black curve, labelled 'A').

The experimental results in Fig. 5 clearly show that, for small detunings, the lower cw state is metastable: it is quickly invaded by the upper state, with the SWs moving towards each other $[V<0$, as in Fig. 4(a)]. As the detuning increases, the SW speed $V$ decreases, and crosses zero at about $\Delta_{\mathrm{M}} \approx 10.4$ (average value for the six independent realisations). For larger detunings, we see clearly how it is now the upper cw state that is metastable, shrinking at the expense of the lower state (i.e. $V>0$ ). The zero-crossing of the SW velocity corresponds, of course, to the Maxwell point for our parameters. In this context, we highlight that the value $\Delta_{\mathrm{M}} \approx 10.4$ extracted from our measurements is in very good agreement with the value obtained from numerical simulations $\left[\Delta_{\mathrm{M}} \approx 10.3\right.$, see Fig. 1(e)]. Numerically calculated SW velocities are also plotted in Fig. 5 (solid black curve, labelled ' $\mathrm{N}$ ') for the full range of detunings considered in the experiment and are found to match the measurements reasonably well.

The SW velocities shown in Fig. 5 were extracted for SWs evolving independently, i.e. without interaction. Of course, departure from purely linear front motion can be expected when the SWs get sufficiently close to each other for their oscillating tails to overlap [44]. Experimental observation of such interaction (and the prospect of eventual interlocking) is, however, hindered by the inherent fluctuations of the system, as well as the small amplitudes of the oscillatory tails that underpin the interactions. Intuitively, one may expect that SW interlocking is most likely to take place close to the Maxwell point, where the SW velocities are already small. Accordingly, we have looked for signa- tures of SW interactions by analysing data acquired for a detuning $\Delta \approx 9.9$ (just below the Maxwell point) over a larger number of round trips. These measurements are presented in Figs 6(a) and (b), again in the form of spacetime diagrams. Data shown in these two panels correspond to two different driving pulses [respectively \#1 and \#3 as labelled in Fig. 3(a)] but were otherwise acquired simultaneously, i.e. for the exact same detuning. Only the driving powers are slightly different as already mentioned. As can be seen, the lower state domain initially contracts, as in Fig. 4(a), but then, instead of merging together, the two SWs slow down and eventually come to a halt, giving rise to a persisting localised dark structure.

The temporal intensity profiles of the observed dark structures are shown in more detail in Figs 6(c) and (d), respectively for each of the two driving pulses considered here. In this plot, we overlay oscilloscope traces corresponding to the last 400 round trips of our measurements, highlighting how the observed structures maintain a constant shape and power level. Unfortunately, the limited $12 \mathrm{GHz}$ bandwidth of our detection system is insufficient to properly resolve the optical dark structures, which according to simulations should not be larger than 25 ps. In this context, we suspect that the difference in the depth of the dark dips registered in Figs 6(c) and (d) is an artefact, and is rather indicative of dark structures with different temporal durations. Indeed, for timescales shorter than the impulse response, the detector simply yields a measurement proportional to the signal energy. As shorter dips lead to a reduced drop in detected energy, one expects them to register as a shallower feature on the oscilloscope.

The results shown in Fig. 6 are indicative of SW interlocking. However, because of the limited memory depth of our oscilloscope, the measurement only encompasses

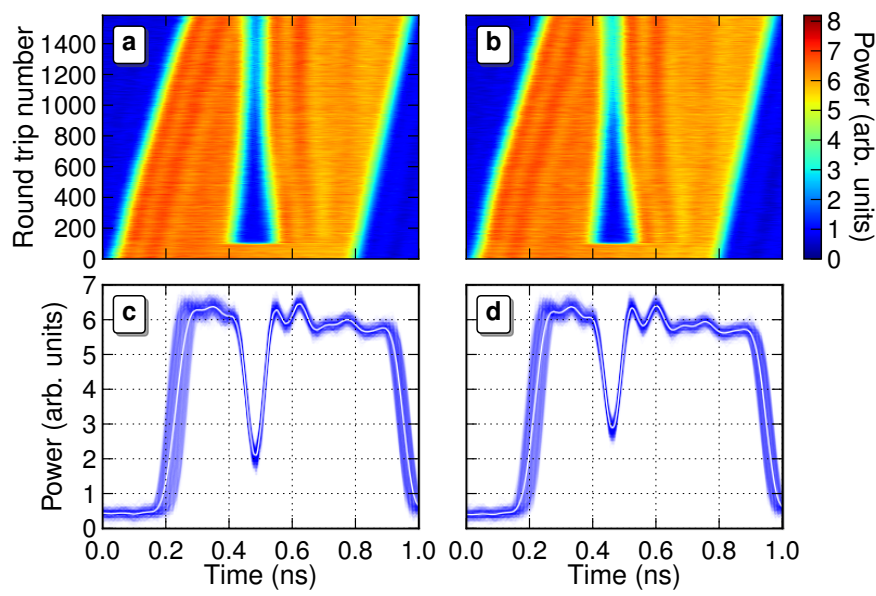

Fig. 6. Experimental measurements showing evidence of SW interlocking, obtained for $\Delta \approx 9.9$. (a, b) Space-time diagrams of the cavity output power corresponding, respectively, to driving pulses \#1 and \#3 as labelled in Fig. 3(a). (c, d) Superpositions of oscilloscope traces corresponding to the last 400 round trips of the data shown in $(\mathrm{a}, \mathrm{b})$. The white lines represent the average of the traces displayed. 

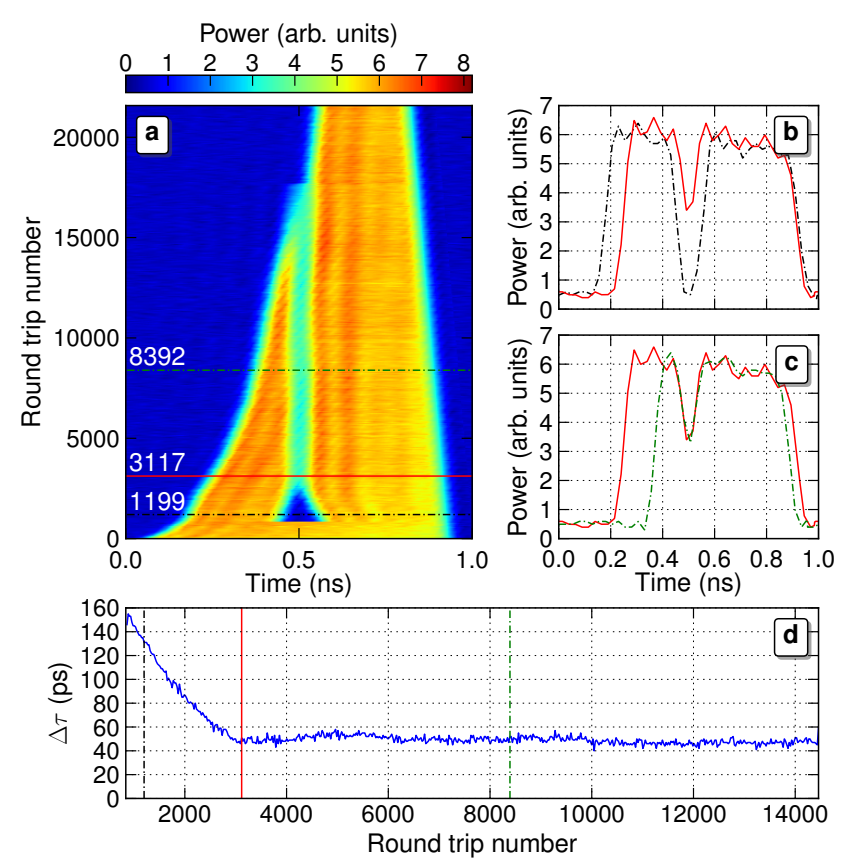

Fig. 7. Experimental results showing the long-term stability of interlocked SWs. The data was obtained for $\Delta \approx 9.4$ with driving pulse \#2. (a) Space-time diagram of the evolution of the temporal power profile at the cavity output. (b, c) Selected snapshots; the colours correspond to the horizontal lines in (a), with the corresponding round trip number as indicated. (d) Corresponding evolution of the separation of the two SWs, $\Delta \tau$.

about 1000 round trips, preventing full conclusions on the long term stability of the dark structures. To gain more convincing evidence, we performed another set of measurements for which we captured the cavity output only once every 23 round trips, leading to a coarser but significantly longer acquisition. The result is presented in Fig. 7. As in Figs 6(a) and (b), we observe an initially shrinking lower state domain, and the associated SWs eventually interlock around the 3000th round trip. This behaviour can be observed in more details with the selected snapshots plotted in Figs 7(b) and (c), and also in the evolution of the separation between the two SWs [Fig. 7(d)], which presents a stage of linear decrease followed by a characteristic plateau. The formed dark structure is seen to persist unchanged for more than 10000 round trips, which corresponds to almost 2000 photon lifetimes. Eventually, around round trip \#17500, the dark structure is destroyed as it collides with another SW that has emerged spontaneously from the leading edge of the driving pulse. Nevertheless, the fact that the dark structure persists for almost 2000 photon lifetimes is clear evidence of stable SW interlocking.

\section{Conclusions}

In conclusion, we have experimentally and theoretically investigated the dynamics of SWs in a passive Kerr fibre ring resonator operating in the normal dispersion regime. By locally perturbing the homogeneous intracavity field in a region of cw bistability, we were able to systematically generate SWs and investigate their dynamics. In particular, we measured the velocities of the SWs as a function of the cavity detuning, observing a zero crossing at the point of equal stability (the Maxwell point). Additionally, for parameters sufficiently close to the Maxwell point, we observed clear evidence of interlocking of two SWs and the corresponding formation of persisting dark temporal structures. Evidence for the multistability of the structure width have also been presented, but are limited by the resolution of our detection system. All our experimental results are in good agreement with simulations of the Lugiato-Lefever equation. Our findings help link recent theoretical and numerical works on SWs [44] to the generation of dark pulse Kerr frequency combs observed in normally dispersive microresonator experiments [40], and provide insights on the operation of coherently driven fibre ring resonator in the normal dispersion regime.

\section{Acknowledgements}

We gratefully acknowledge support from the Marsden Fund and the Rutherford Discovery Fellowships of the Royal Society of New Zealand.

\section{Author contribution statement}

All experiments were performed by B. Garbin with the help of S. G. Murdoch and Y. Wang. Numerical simulations were performed by B. Garbin with the help of S. Coen. G.-L. Oppo helped with the theoretical explanation and the connection with the literature. The manuscript was written by M. Erkintalo and B. Garbin, with help from all of the other authors. The project was supervised by S. Coen, S. G. Murdoch, G.-L. Oppo and M. Erkintalo. All authors contributed to the interpretation of data and discussion of results.

\section{References}

1. M. Haelterman, S. Trillo, and S. Wabnitz, "Dissipative modulation instability in a nonlinear dispersive ring cavity," Optics Communications 91, 401-407 (1992). doi: 10.1016/0030-4018(92)90367-Z.

2. S. Coen, M. Haelterman, Ph. Emplit, L. Delage, L. M. Simohamed, and F. Reynaud, "Experimental investigation of the dynamics of a stabilized nonlinear fiber ring resonator," Journal of the Optical Society of America B 15, 2283-2293 (1998). doi: 10.1364/JOSAB.15.002283.

3. S. Coen and M. Haelterman, "Continuous-wave ultrahighrepetition-rate pulse-train generation through modulational instability in a passive fiber cavity," Optics Letters 26, 39-41 (2001). doi: 10.1364/OL.26.000039. 
4. F. Leo, S. Coen, P. Kockaert, S.-P. Gorza, Ph. Emplit, and M. Haelterman, "Temporal cavity solitons in onedimensional Kerr media as bits in an all-optical buffer," Nature Photonics 4, 471-476 (2010). doi: 10.1038/nphoton.2010.120.

5. F. Leo, L. Gelens, Ph. Emplit, M. Haelterman, and S. Coen, "Dynamics of one-dimensional Kerr cavity solitons," Optics Express 21, 9180-9191 (2013). doi: 10.1364/OE.21.009180.

6. J. K. Jang, M. Erkintalo, S. G. Murdoch, and S. Coen, "Ultraweak long-range interactions of solitons observed over astronomical distances," Nature Photonics 7, 657-663 (2013). doi: 10.1038/nphoton.2013.157.

7. J. K. Jang, M. Erkintalo, S. Coen, and S. G. Murdoch, "Temporal tweezing of light through the trapping and manipulation of temporal cavity solitons," Nature Communications 6, 7370 (2015). doi: 10.1038/ncomms8370.

8. F. Copie, M. Conforti, A. Kudlinski, A. Mussot, and S. Trillo, "Competing Turing and Faraday instabilities in longitudinally modulated passive resonators," Physical Review Letters 116, 143901/1-5 (2016). doi: 10.1103/PhysRevLett.116.143901.

9. J. K. Jang, M. Erkintalo, J. Schröder, B. J. Eggleton, S. G. Murdoch, and S. Coen, "All-optical buffer based on temporal cavity solitons operating at $10 \mathrm{~Gb} / \mathrm{s}$," Optics Letters 41, 4526-4529 (2016). doi: 10.1364/OL.41.004526.

10. P. Del'Haye, A. Schliesser, O. Arcizet, T. Wilken, R. Holzwarth, and T. J. Kippenberg, "Optical frequency comb generation from a monolithic microresonator," Nature 450, 1214-1217 (2007). doi: 10.1038/nature06401.

11. T. J. Kippenberg, R. Holzwarth, and S. A. Diddams, "Microresonator-based optical frequency combs," Science 332, 555-559 (2011). doi: 10.1126/science.1193968.

12. A. A. Savchenkov, A. B. Matsko, W. Liang, V. S. Ilchenko, D. Seidel, and L. Maleki, "Kerr combs with selectable central frequency," Nature Photonics 5, 293-296 (2011). doi: 10.1038/nphoton.2011.50.

13. F. Ferdous, H. Miao, D. E. Leaird, K. Srinivasan, J. Wang, L. Chen, L. T. Varghese, and A. M. Weiner, "Spectral line-by-line pulse shaping of on-chip microresonator frequency combs," Nature Photonics 5, 770-776 (2011). doi: 10.1038/nphoton.2011.255.

14. T. Herr, V. Brasch, J. D. Jost, C. Y. Wang, N. M. Kondratiev, M. L. Gorodetsky, and T. J. Kippenberg, "Temporal solitons in optical microresonators," Nature Photonics 8, 145-152 (2014). doi: 10.1038/nphoton.2013.343.

15. R. Henriet, G. Lin, A. Coillet, M. Jacquot, L. Furfaro, L. Larger, and Y. K. Chembo, "Kerr optical frequency comb generation in strontium fluoride whispering-gallery mode resonators with billion quality factor," Optics Letters 40, 1567-1570 (2015). doi: 10.1364/OL.40.001567.

16. X. Yi, Q.-F. Yang, K. Y. Yang, M.-G. Suh, and K. Vahala, "Soliton frequency comb at microwave rates in a high-Q silica microresonator," Optica 2, 1078-1085 (2015). doi: 10.1364/OPTICA.2.001078.

17. C. Joshi, J. K. Jang, K. Luke, X. Ji, S. A. Miller, A. Klenner, Y. Okawachi, M. Lipson, and A. L. Gaeta, "Thermally controlled comb generation and soliton modelocking in microresonators," Optics Letters 41, 2565-2568 (2016). doi: 10.1364/OL.41.002565.

18. K. E. Webb, M. Erkintalo, S. Coen, and S. G. Murdoch, "Experimental observation of coherent cavity soliton frequency combs in silica microspheres," Optics Letters 41, 4613-4616 (2016). doi: 10.1364/OL.41.004613.
19. J. Pfeifle, V. Brasch, M. Lauermann, Y. Yu, D. Wegner, T. Herr, K. Hartinger, P. Schindler, J. Li, D. Hillerkuss, R. Schmogrow, C. Weimann, R. Holzwarth, W. Freude, J. Leuthold, T. J. Kippenberg, and C. Koos, "Coherent terabit communications with microresonator Kerr frequency combs," Nature Photonics 8, 375-380 (2014). doi: 10.1038/nphoton.2014.57.

20. J. Pfeifle, A. Coillet, R. Henriet, K. Saleh, P. Schindler, C. Weimann, W. Freude, I. V. Balakireva, L. Larger, C. Koos, and Y. K. Chembo, "Optimally coherent Kerr combs generated with crystalline whispering gallery mode resonators for ultrahigh capacity fiber communications," Physical Review Letters 114, 093902/1-5 (2015). doi: 10.1103/PhysRevLett.114.093902.

21. M.-G. Suh, Q.-F. Yang, K. Y. Yang, X. Yi, and K. J. Vahala, "Microresonator soliton dual-comb spectroscopy," Science 354, 600-603 (2016). doi: 10.1126/science.aah6516.

22. A. Dutt, C. Joshi, X. Ji, J. Cardenas, Y. Okawachi, K. Luke, A. L. Gaeta, and M. Lipson, "On-chip dual comb source for spectroscopy," arXiv 1611.07673 (2016).

23. S. Coen, H. G. Randle, T. Sylvestre, and M. Erkintalo, "Modeling of octave-spanning Kerr frequency combs using a generalized mean-field Lugiato-Lefever model," Optics Letters 38, 37-39 (2013). doi: 10.1364/OL.38.000037.

24. S. Coen and M. Erkintalo, "Universal scaling laws of Kerr frequency combs," Optics Letters 38, 1790-1792 (2013). doi: 10.1364/OL.38.001790.

25. Y. K. Chembo and C. R. Menyuk, "Spatiotemporal Lugiato-Lefever formalism for Kerr-comb generation in whispering-gallery-mode resonators," Physical Review A 87,053852/1-4 (2013). doi: 10.1103/PhysRevA.87.053852.

26. L. A. Lugiato and R. Lefever, "Spatial dissipative structures in passive optical systems," Physical Review Letters 58, 2209-2211 (1987). doi: 10.1103/PhysRevLett.58.2209.

27. G. J. Morales and Y. C. Lee, "Ponderomotive-force effects in a nonuniform plasma," Phys. Rev. Lett. 33, 1016-1019 (1974). doi: 10.1103/PhysRevLett.33.1016.

28. K. Nozaki and N. Bekki, "Solitons as attractors of a forced dissipative nonlinear schrdinger equation," Physics Letters A 102, 383 - 386 (1984). doi: http://dx.doi.org/10.1016/0375-9601(84)91060-0.

29. K. Nozaki and N. Bekki, "Low-dimensional chaos in a driven damped nonlinear schrdinger equation," Physica D: Nonlinear Phenomena 21, 381 - 393 (1986). doi: http://dx.doi.org/10.1016/0167-2789(86)90012-6.

30. D. J. Kaup and A. C. Newell, "Theory of nonlinear oscillating dipolar excitations in one-dimensional condensates," Phys. Rev. B 18, 5162-5167 (1978). doi: 10.1103/PhysRevB.18.5162.

31. K. Luo, J. K. Jang, S. Coen, S. G. Murdoch, and M. Erkintalo, "Spontaneous creation and annihilation of temporal cavity solitons in a coherently driven passive fiber resonator," Optics Letters 40, 3735-3738 (2015). doi: 10.1364/OL.40.003735.

32. J. K. Jang, M. Erkintalo, K. Luo, G.-L. Oppo, S. Coen, and S. G. Murdoch, "Controlled merging and annihilation of localised dissipative structures in an AC-driven damped nonlinear Schrödinger system," New Journal of Physics 18, 033034/1-7 (2016). doi: 10.1088/1367-2630/18/3/033034.

33. M. Anderson, F. Leo, S. Coen, M. Erkintalo, and S. G. Murdoch, "Observations of spatiotemporal instabilities of temporal cavity solitons," Optica 3, 1071-1074 (2016). doi: 10.1364/OPTICA.3.001071. 
34. S. Coen and M. Haelterman, "Modulational instability induced by cavity boundary conditions in a normally dispersive optical fiber," Physical Review Letters 79, 4139-4142 (1997). doi: 10.1103/PhysRevLett.79.4139.

35. S. Coen and M. Haelterman, "Competition between modulational instability and switching in optical bistability," Optics Letters 24, 80-82 (1999). doi: 10.1364/OL.24.000080.

36. A. B. Matsko, A. A. Savchenkov, and L. Maleki, "Normal group-velocity dispersion Kerr frequency comb," Optics Letters 37, 43-45 (2012). doi: 10.1364/OL.37.000043.

37. C. Godey, I. V. Balakireva, A. Coillet, and Y. K. Chembo, "Stability analysis of the spatiotemporal Lugiato-Lefever model for Kerr optical frequency combs in the anomalous and normal dispersion regimes," Physical Review A 89, 063814/1-21 (2014). doi: 10.1103/PhysRevA.89.063814.

38. A. Coillet, I. Balakireva, R. Henriet, K. Saleh, L. Larger, J. M. Dudley, C. Menyuk, and Y. Chembo, "Azimuthal Turing patterns, bright and dark cavity solitons in Kerr combs generated with whispering-gallery-mode resonators," IEEE Photonics Journal 5, 6100409/1-9 (2013). doi: 10.1109/JPHOT.2013.2277882.

39. W. Liang, A. A. Savchenkov, V. S. Ilchenko, D. Eliyahu, D. Seidel, A. B. Matsko, and L. Maleki, "Generation of a coherent near-infrared Kerr frequency comb in a monolithic microresonator with normal GVD," Optics Letters 39, 2920-2923 (2014). doi: 10.1364/OL.39.002920.

40. X. Xue, Y. Xuan, Y. Liu, P.-H. Wang, S. Chen, J. Wang, D. E. Leaird, M. Qi, and A. M. Weiner, "Mode-locked dark pulse Kerr combs in normal-dispersion microresonators," Nature Photonics 9, 594-600 (2015). doi: 10.1038/nphoton.2015.137.

41. X. Xue, F. Leo, Y. Xuan, J. A. Jaramillo-Villegas, P.H. Wang, D. E. Leaird, M. Erkintalo, M. Qi, and A. M. Weiner, "Second-harmonic assisted four-wave mixing in chip-based microresonator frequency comb generation," arXiv 1607.02711 (2016).

42. M. Tlidi, A. Mussot, E. Louvergneaux, G. Kozyreff, A. G. Vladimirov, and M. Taki, "Control and removal of modulational instabilities in low-dispersion photonic crystal fiber cavities," Optics Letters 32, 662-664 (2007). doi: 10.1364/OL.32.000662.

43. V. E. Lobanov, G. Lihachev, T. J. Kippenberg, and M. L. Gorodetsky, "Frequency combs and platicons in optical microresonators with normal GVD," Optics Express 23, 7713-7721 (2015). doi: 10.1364/OE.23.007713.

44. P. Parra-Rivas, D. Gomila, E. Knobloch, S. Coen, and L. Gelens, "Origin and stability of dark pulse Kerr combs in normal dispersion resonators," Optics Letters 41, 24022405 (2016). doi: 10.1364/OL.41.002402.

45. N. N. Rozanov, V. E. Semenov, and G. V. Khodova, "Transverse structure of a field in nonlinear bistable interferometers. I. Switching waves and steady-state profiles," Soviet Journal of Quantum Electronics 12, 193-197 (1982). doi: 10.1070/QE1982v012n02ABEH005474.

46. V. Petrov, Q. Ouyang, and H. L. Swinney, "Resonant pattern formation in achemical system," Nature 388, 655-657 (1997). doi: 10.1038/41732.

47. S. P. Dawson, J. Keizer, and J. E. Pearson, "Fire-diffusefire model of dynamics of intracellular calcium waves," Proceedings of the National Academy of Sciences of the United States of America 96, 6060-6063 (1999). doi: 10.1073/pnas.96.11.6060.
48. A. H. Eschenfelder, Magnetic Bubble Technology, number 14 in Springer Series in Solid-State Sciences (1981).

49. J. E. Macías, M. G. Clerc, C. Falcón, and M. A. GarcíaNustes, "Spatially modulated kinks in shallow granular layers," Physical Review E 88, 020201/1-4 (2013). doi: 10.1103/PhysRevE.88.020201.

50. Y. Pomeau, "Front motion, metastability and subcritical bifurcations in hydrodynamics," Physica D: Nonlinear Phenomena 23, 3-11 (1986). doi: 10.1016/01672789(86)90104-1.

51. M. G. Clerc, D. Escaff, and V. M. Kenkre, "Patterns and localized structures in population dynamics," Physical Review E 72, 056217/1-5 (2005). doi: 10.1103/PhysRevE.72.056217.

52. F. A. Williams, Combustion Theory, Addison Wesley (1965).

53. S. Coen, M. Tlidi, Ph. Emplit, and M. Haelterman, "Convection versus dispersion in optical bistability," Physical Review Letters 83, 2328-2331 (1999). doi: 10.1103/PhysRevLett.83.2328.

54. G.-L. Oppo, A. J. Scroggie, and W. J. Firth, "From domain walls to localized structures in degenerate optical parametric oscillators," Journal of Optics B: Quantum and Semiclassical Optics 1, 133-138 (1999). doi: 10.1088/14644266/1/1/024.

55. I. Ganne, G. Slekys, I. Sagnes, and R. Kuszelewicz, "Optical switching waves in III-V semiconductor microresonators," Phys. Rev. B 63, 075318 (2001). doi: 10.1103/PhysRevB.63.075318.

56. D. Gomila, P. Colet, G.-L. Oppo, and M. San Miguel, "Stable droplets and growth laws close to the modulational instability of a domain wall," Physical Review Letters 87, 194101/1-4 (2001). doi: 10.1103/PhysRevLett.87.194101.

57. S. Residori, "Patterns, fronts and structures in a liquidcrystal-light-valve with optical feedback," Physics Reports 416, 201-272 (2005). doi: 10.1016/j.physrep.2005.06.004.

58. F. Marino, G. Giacomelli, and S. Barland, "Front pinning and localized states analogues in long-delayed bistable systems," Phys. Rev. Lett. 112, 103901 (2014). doi: 10.1103/PhysRevLett.112.103901.

59. V. Odent, M. Tlidi, M. G. Clerc, P. Glorieux, and E. Louvergneaux, "Experimental observation of front propagation in a negatively diffractive inhomogeneous Kerr cavity," Phys. Rev. A 90, 011806 (2014). doi: 10.1103/PhysRevA.90.011806.

60. F. Gustave, L. Columbo, G. Tissoni, M. Brambilla, F. Prati, and S. Barland, "Phase solitons and domain dynamics in an optically injected semiconductor laser," Phys. Rev. A 93, 063824 (2016). doi: 10.1103/PhysRevA.93.063824.

61. W. Firth and I. Galbraith, "Diffusive transverse coupling of bistable elements - Switching waves and crosstalk," IEEE Journal of Quantum Electronics 21, 1399-1403 (1985). doi: 10.1109/JQE.1985.1072842.

62. T. Ackemann, W. J. Firth, and G.-L. Oppo, "Chapter 6: Fundamentals and applications of spatial dissipative solitons in photonic devices," Advances in Atomic, Molecular and Optical Physics 57, 323-421 (2009).

63. B. A. Malomed, "Bound states of envelope solitons," Physical Review E 47, 2874-2880 (1993). doi: 10.1103/PhysRevE.47.2874.

64. B. Schäpers, M. Feldmann, T. Ackemann, and W. Lange, "Interaction of localized structures in an optical pattern- 
forming system," Physical Review Letters 85, 748-751 (2000). doi: 10.1103/PhysRevLett.85.748.

65. N. N. Rosanov and G. V. Khodova, "Diffractive autosolitons in nonlinear interferometers," Journal of the Optical Society of America B 7, 1057-1065 (1990). doi: 10.1364/JOSAB.7.001057.

66. R. Gallego, M. San Miguel, and R. Toral, "Self-similar domain growth, localized structures, and labyrinthine patterns in vectorial Kerr resonators," Physical Review E 61, 2241-2244 (2000). doi: 10.1103/PhysRevE.61.2241.

67. G.-L. Oppo, A. J. Scroggie, and W. J. Firth, "Characterization, dynamics and stabilization of diffractive domain walls and dark ring cavity solitons in parametric oscillators," Physical Review E 63, 066209/1-16 (2001). doi: 10.1103/PhysRevE.63.066209.

68. M. Pesch, W. Lange, D. Gomila, T. Ackemann, W. J. Firth, and G.-L. Oppo, "Two-dimensional front dynamics and spatial solitons in a nonlinear optical system," Physical Review Letters 99, 153902 (2007). doi: 10.1103/PhysRevLett.99.153902.

69. Y.-P. Ma, J. Burke, and E. Knobloch, "Defect-mediated snaking: A new growth mechanism for localized structures," Physica D: Nonlinear Phenomena 239, 1867-1883 (2010). doi: 10.1016/j.physd.2010.06.014.

70. S. Coulibaly, M. Taki, and M. Tlidi, "Universal power law for front propagation in all fiber resonators," Opt. Express 22, 483-489 (2014). doi: 10.1364/OE.22.000483.

71. P. Parra-Rivas, E. Knobloch, D. Gomila, and L. Gelens, "Dark solitons in the Lugiato-Lefever equation with normal dispersion," Physical Review A 93, 063839/1-17 (2016). doi: 10.1103/PhysRevA.93.063839.

72. I. Rabbiosi, A. J. Scroggie, and G.-L. Oppo, "Stochastic resonance in the presence of spatially localized structures," Physical Review E 68, 036602/1-10 (2003). doi: 10.1103/PhysRevE.68.036602.

73. M. H. Anderson, Y. Wang, F. Leo, S. Coen, M. Erkintalo, and S. G. Murdoch, "Super cavity solitons and the coexistence of multiple nonlinear states in a tristable passive Kerr resonator," arXiv 1702.00782 (2017). 